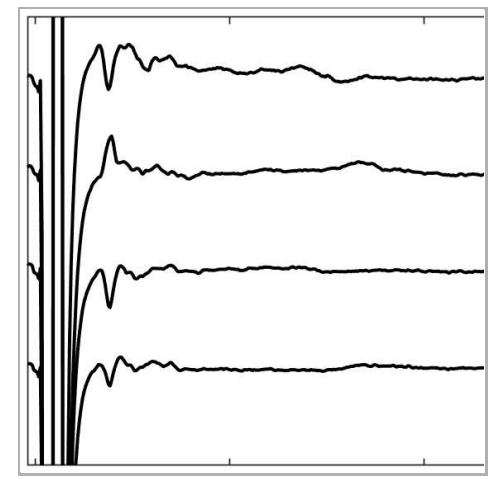

AUG 01, 2021

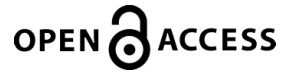

DOI:

dx.doi.org/10.17504/protocol s.io.bkeyktfw

Protocol Citation: Evan N Nicolai, Kip Ludwig 2021. Vagus Nerve Stimulation Evoked Electroneurography and Electromyography Recordings in Swine. protocols.io

https://dx.doi.org/10.17504/p rotocols.io.bkeyktfw

MANUSCRIPT CITATION:

Nicolai, Evan N et al. "Sources of off-target effects of vagus nerve stimulation using the helical clinical lead in domestic pigs." Journal of neural engineering vol. 17,4 046017 24 Jul. 2020,

doi:10.1088/1741-

2552/ab9db8

License: This is an open access protocol distributed under the terms of the Creative Commons Attribution License, which permits unrestricted use, distribution, and reproduction in any medium, provided the original author and source are credited

Protocol status: Working We use this protocol and it's working

\section{(3) Vagus Nerve Stimulation Evoked Electroneurography and Electromyography Recordings in Swine}

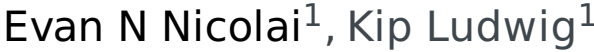 \\ ${ }^{1}$ University of Wisconsin - Madison \\ SPARC \\ Tech. support email: info@neuinfo.org
}

\section{Evan N Nicolai}

\section{ABSTRACT}

This protocol was used to collect data now published in the Journal of Neural Engineering, Sources of off-target effects of vagus nerve stimulation using the helical clinical lead in domestic pigs, see Metadata for citation. Additionally, the dataset is publicly available on Pennsieve 10.26275/qcuk-a8ty.

\section{IMAGE ATTRIBUTION}

Data collected by Evan Nicolai.

\section{GUIDELINES}

DISCLAIMER - FOR INFORMATIONAL PURPOSES ONLY; USE AT YOUR OWN RISK

The protocol content here is for informational purposes only and does not constitute legal, medical, clinical, or safety advice, or otherwise; content added to protocols.io is not peer reviewed and may not have undergone a formal approval of any kind. Information presented in this protocol should not substitute for independent professional judgment, advice, diagnosis, or treatment. Any action you take or refrain from taking using or relying upon the information presented here is strictly at your own risk. You agree that neither the Company nor any of the authors, contributors, administrators, or anyone else associated with protocols.io, can be held responsible for your use of the information contained in or linked to this protocol or any of our Sites/Apps and Services.

Created: Aug 28, 2020 
PROTOCOL integer ID: 41144

Keywords: electrophysiology, electromyography, swine, vns
Stimulation Electrode (PerreniaFLEX Model 304, 2 mm inner diameter, LivaNova, Houston, TX)

Recording Electrodes (made in-house, Longitudinal Intrafascicular Electrodes) components:

$75 \mu \mathrm{m}$ outer diameter PFA-coated platinum wire (AM-Systems, Sequim, WA) suture needle (Item No. 12050-03, Fine Science Tools, Foster City, CA) insulated copper extension wire with touchproof connector (441 connector with wire, Plastics1, Roanoke, VA)

Stimulation and Recording Devices:

Tucker Davis Technologies system (Alachua, FL; W8, IZ2MH, RZ5D, RZ6, PZ5, and SBox units)

Data Analysis:

Matlab R2018b (Mathworks, Natick, MA)

\section{Animal Preparation, Surgical Dissection, and Placement of}

Note

Consult your institution's IACUC before attempting any of the following.

What we did (approved by the Mayo Clinic IACUC):

Weigh pig, and inject (I.M.) the pig with a mixture of telazol $(6 \mathrm{mg} / \mathrm{kg})$, xylazine $(2 \mathrm{mg} / \mathrm{kg})$, and glycopyrrolate $(0.006 \mathrm{mg} / \mathrm{kg})$. Intubate the pig and anesthetize using isoflurane gas $(1.5-3 \%$ in room air).

2 Position the pig in supine position with neck extended, and make a single incision through the skin and superficial fat layers between the mandible and sternal notch using a cautery. Use blunt dissection to expose the carotid sheath and isolate the vagus nerve from the carotid artery.

3 Use blunt dissection to locate the thyroid cartilage and identify the cricothyroid and cricoarytenoid muscles. 
4 Use blunt dissection to locate the superior and recurrent laryngeal branches of the vagus nerve.

The superior branch inserts into the vagus trunk near the nodose ganglion and projects towards the thyroid cartilage. The nodose ganglion should be near the carotid bifurcation of the carotid artery, and will look like an enlargement (diameter) along the length of the nerve. Place a loose suture tie around the branch to locate the branch easily later.

The recurrent branch inserts into the vagus trunk within the thorax, and thus cannot be identified within the cervical surgical window for the left or right side vagus trunk. The branch must be located along the length of the trachea projecting towards the thyroid cartilage. Place a loose suture tie around the branch to locate the branch easily later.

5 Use blunt dissection to expose as much length of the vagus trunk as possible. Ideally at least 8 $\mathrm{cm}$ from the superior laryngeal branch insertion point to a more caudal location.

6 Place stimulation electrode (we used the LivaNova PerrenialFLEX Model 304, 2 mm inner diameter) such that both cathode and anode are caudal to the superior laryngeal branch on the main vagus trunk.

7 Place electrophysiology (electroneurography, ENG) recording electrodes (we used 50 micron diameter bare wire intrafascicular electrodes made in-house) such that the distance between stimulation and recording electrodes is maximized. Given that compound action potentials have been reported to have conduction speeds as high as $120 \mathrm{~m} / \mathrm{s}$ (A-alpha fibers), greater distance between stimulation and recording electrodes will more clearly temporally separate the compound action potential signals from the stimulation artifact.

8 Place electromyography (EMG) recording electrodes (we used 200 micron diameter stainless steel wires with sharpened tips) into the cricothyroid and cricoarytenoid muscles. Two electrodes per muscle should be placed, and care should be taken that two leads do not touch.

\section{Stimulation and Recording Equipment Setup}

9 Stimulation system. We used the Tucker Davis Technologies (TDT) IZ2MH as well as the S-Box to connect leads. This stimulator is optically isolated from the recording system. Additionally, the system is battery powered.

Connect the stimulator to the two stimulation electrode contacts on the vagus nerve.

Set the stimulation waveform to be symmetric biphasic and have a period of 33.3 milliseconds 
(30 hz stimulation), pulse width 200 microseconds, interpulse duration 0 microseconds, and stimulate for 30 seconds total ( 900 pulses delivered). Stimulation should be set to constant current mode and stimulations between 50 microamps and 3000 microamps will be used.

NOTE: We have found that using a period of 40 milliseconds ( $25 \mathrm{hz}$ stimulation) is helpful to avoid $60 \mathrm{hz}$ line noise from persisting into stimulation triggered averages or medians (see section on data analysis).

10 Recording system. We used the Tucker Davis Technologies (TDT) W8, RZ5D, RZ6, PZ5, and S-Box units. The recording system is optically isolated from the stimulation system. Additionally, the system is connected to an uninterruptible power supply (UPS).

Using leads from the S-Box, connect to the recording electrodes. All nerve electrodes except one and EMG electrodes should be connected to the numbered leads on the S-Box. The last nerve electrode is the reference electrode for all recording electrodes. Differential recordings for EMG will be created in digital processing (one electrode recording minus the other, per muscle group, see data analysis section).

Place a large diameter needle in to the dermis tissue of the surgical site, being careful not to puncture any vessels. Connect the ground lead for the S-Box to this needle.

11 Record some data and check noise levels. Noise should be less than 10 microvolts peak to peak.

Given we performed these studies in a surgical suite without a faraday cage, additional grounding was performed to reduce noise levels. Likewise, some items unnecessary to animal care were removed or unplugged.

Use wires to attach to large metal items in the surgical suite. At the end of the wire should be a banana plug that will go into the ground socket of an outlet. All large metal objects like metal tables and IV poles should be grounded together.

We also unplugged devices like the cautery and water heater pump. Warmed blankets were used to keep the pig warm after recordings began.

12 Deliver 30 seconds of stimulation using the parameters specified above at $1 \mathrm{~mA}$ amplitude.

Watch for neck muscle contraction. If neck muscle contraction is not obvious, physically adjust the stimulation electrode and check for conformance to the nerve. Also check wire connections. Possibly try higher stimulation amplitudes, but something is likely wrong with conformance of the electrode if neck contraction is not obtainable below $1 \mathrm{~mA}$.

Deliver 30 seconds of stimulation using the parameters specified above at $2 \mathrm{~mA}$ amplitude, then up to $3 \mathrm{~mA}$ in $0.25 \mathrm{~mA}$ increments.

Watch for changes in heart rate (bradycardia), the hering breuer reflex, and adverse reactions. 
Heart rate changes should be obtained near $2 \mathrm{~mA}$ and the hering breuer reflex should be obtained between 2 and $3 \mathrm{~mA}$. Again, attempt repositioning the stimulation electrode or check connections if heart rate changes or the hering breuer response is not obtained below $3 \mathrm{~mA}$.

NOTE: Isoflurane at levels above $2 \%$ will blunt vagus nerve stimulation induced heart rate changes. Therefore, if higher levels of isoflurane ( $>1.5 \%)$ were used during surgery or are required to keep the animal anesthetized at any point, heart rate changes may not be obtainable for some period of time.

The hering breuer reflex in an anesthetized, intubated swine will manifest as abdomen contractions. The end tidal trace will be changed from a smooth plateau to two or more jagged plateaus with each breath.

Adverse reactions include gross bodily movements other than the abdomen, extreme changes in heart rate $(>20 \%)$, and asystole. If such reactions occur, write down the stimulation amplitude that is $0.25 \mathrm{~mA}$ below the one that produced the adverse reaction. This amplitude will be used for verifying responses later. In most cases, we were able to apply $3 \mathrm{~mA}$ which was the limit set across all animals for our studies.

\section{Experiments}

13 While recording electroneurography (ENG) and electromyography (EMG) data, deliver stimulations between 50 and 3000 uA (or the limit found in the previous section), 30 seconds each, in a randomized order with at least 1 minute rest time between each stimulation. We typically delivered the following: $50,100,150,200,250,300,400,500,750,1000,1500,2000$, 2500 , and $3000 \mathrm{uA}$.

If desired, switch the stimulation anode and cathode and collect the same set of data.

14 To temporarily remove EMG contamination from the ENG recordings, give a bolus of vecuronium $(0.1 \mathrm{mg} / \mathrm{kg})$ followed by a continuous drip of vecuronium ( $3 \mathrm{mg} / \mathrm{kg}$ per hour).

Before recording data, stimulate at $1 \mathrm{~mA}$ several times following the bolus injection until neck muscle contraction is no longer occurring. Additionally, if real time analysis are available, check the EMG traces for activity.

Repeat the set of stimulations performed in step 13.

15 To selectively remove (and verify the source of) EMG components that are caused by the recurrent and superior laryngeal branches, record before and after cutting each branch (one at a time).

NOTE: If vecuronium was used, ensure that the effect has subsided before cutting the branches. Usually, at least 15 minutes is required after stopping the vecuronium drip. 
Use $1 \mathrm{~mA}$ stimulations to check for neck muscle contraction following vecuronium drip cessation. The goal of this step is to determine which signals in the EMG are caused by each branch, so recordings of each contraction must occur prior to each transection.

This part of the experiment would look something like this:

1. Perform set of stimulations while recording before transecting branches. We usually abbreviated the set of stimulations to 1,2, and $3 \mathrm{~mA}$ in a random order.

2. Transect either the recurrent or superior laryngeal branch (remember the black suture tie to help locate).

3. Perform stimulations from 1.

4. Transect the other branch.

5. Perform the stimulations from 1.

16 To verify ENG signals after removing the EMG signals, record before and after cutting the vagus nerve trunk between the stimulation and recording electrodes.

Deliver a $3 \mathrm{~mA}$ stimulation (or the highest that can be delivered without adverse effect), and check for neck muscle contraction. If any contraction is obtained, or any EMG signals are obtained, double check that the branches were successfully transected, as well as check for additional branches. Occasionally, two branches were found at the location of the superior laryngeal branch. Likewise, ensure the that the cut end of the superior laryngeal branch leading to the muscle is not too close to the stimulation electrode since the cut end can be activated via current escape at a distance. Keep adjusting until all EMG signals or neck contractions are not obtained in response to this high stimulation amplitude.

Perform the set of stimulations used to verify the EMG signals, then transect the vagus nerve trunk between the stimulation and recording electrodes, and finally perform the set of stimulations again.

\section{Data Analysis}

17 We performed signal processing using Matlab 2018b.

Tucker Davis Technologies (TDT) has Matlab scripts available to download that converts TDT data type to .mat.

18 Filter all ENG and EMG data using a 400 sample median high-pass filter ( $\mathrm{y}=\mathrm{x}$ - medfilt1(x)), followed by a gaussian low-pass filter (gaussfiltcoef and filtfilt) with corner frequency of $5 \mathrm{kHz}$.

19 For each pair of EMG electrode recordings, one should be subtracted from the other to form a differential signal for each muscle group. 
20 Using the stimulation artifact, or the timing of the stimulation pulse which is saved when stimulating using the TDT system, create stimulus triggered averages or medians of each ENG and EMG channel in response to each stimulation applied.

21 Compare ENG signals before and after transection of the recurrent and superior laryngeal branches, as well as before and after administration of vecuronium.

Signals that disappear after transection or vecuronium are artifacts caused by contraction of the neck muscles. Signals that remain are possibly compound action potentials.

Likewise, the same comparisons can be used to identify EMG signals caused by activation of the recurrent laryngeal branch and superior laryngeal branch, respectively.

Compare ENG signals before and after transection of the main vagus trunk.

Signals that disappear after vagus trunk transection are compound action potentials. Importantly, the superior laryngeal and recurrent laryngeal branches must have been transected before this comparison can be made.

22 Compare ENG and EMG signals in response to each stimulation amplitude to determine threshold for activation of different compound action potentials and EMG components.

EMG components can be identified using transections of the superior laryngeal and recurrent laryngeal branches, or by latency since the superior laryngeal is a shorter pathway (therefore, shorter post-stimulus latency).

ENG compound action potentials can be identified by previously reported findings in the literature.

23 For additional information, and presentation of our findings, please see:

Nicolai, Evan $\mathrm{N}$ et al. "Sources of off-target effects of vagus nerve stimulation using the helical clinical lead in domestic pigs." Journal of neural engineeringvol. 17,4 046017. 24 Jul. 2020, doi:10.1088/1741-2552/ab9db8 